\title{
Association between MDR1 gene polymorphisms and the risk of Crohn's disease in a cohort of Algerian pediatric patients
}

\author{
Amira Bouzidi', Hamida Mesbah-Amroun', Aziza Boukercha', Fadila Benhassine², Réda Belboueb³, Karima Berkouk', \\ Wassila Messadi ${ }^{5}$ and Chafia Touil-Boukoffa ${ }^{1}$
}

\begin{abstract}
BACKGROUND: The multi-drug resistance gene (MDR1) has raised increasing interest as a susceptibility gene for Crohn's disease (CD). The role of MDR1 single-nucleotide polymorphisms (SNPs) in the predisposition and behavior of CD in the pediatric population is still elusive. Here, we investigated whether SNPs in MDR1 are associated with CD in Algerian pediatric patients.
\end{abstract}

METHODS: A case-control study was conducted enrolling 47 pediatric CD patients and 100 controls. All subjects were genotyped for the most common MDR1 SNPs (C3434T, C1236T, and G2677A/T) using PCR-RFLP method. We also explored the association between polymorphisms and clinical sub-phenotypes. RESULTS: We have detected no significant association of C3435T SNP and pediatric CD. However, we observed a significantly higher frequency of the risk alleles, 1236T and 2677T/A among the CD patients compared to controls. Moreover, the risk allele 1236T was associated to a higher risk for resective surgery.

CONCLUSION: Our data suggest that the C1236T and G2677A/T SNPs in the MDR1 gene are associated with CD and the C1236T risk allele with a more severe course of disease in Algerian pediatric patients. Further analysis using larger patients group and functional studies would be interesting to elucidate the role of MDR1 gene in pediatric CD.

\footnotetext{
nflammatory bowel disease (IBD) is a disorder characterized by chronic inflammation of the gastrointestinal tract. There are two common clinical subtypes, Crohn's disease (CD) and ulcerative colitis (UC). CD can affect any part of the intestine and is associated with discontinuous transmural lesions of the gut wall (1). CD affects both adult and children with the peak age range of diagnosis in the second and third decades of life. In childhood, rates of $\mathrm{CD}$ increase from the first year of life, with highest rates in teenage years (2).

The etiology of CD involves environmental and genetic factors that lead to dysfunction of the epithelial barrier with consequent deregulation of the mucosal immune system and responses to gut microbiota (1).
}

Over the last $15 \mathrm{y}$, linkage analysis, positional candidate gene studies, and genome-wide association studies (GWAS) provided the most convincing evidence of a genetic link, identifying a number of susceptibility genes for $\mathrm{CD}$. In particular, the NOD2/CARD15 (3,4), IL23R (5), ATG16L1 (6) genes have been widely implicated in the pathogenesis of IBD in different geographical populations. Numerous data have shown that the underlying cause of susceptibility to $\mathrm{CD}$ is a complex interplay between autophagy, innate immunity, and adaptive immunity. Most genetic analyses in $\mathrm{CD}$ have been performed in patients with adult-onset disease (7). Pediatric CD, however, has unique characteristics of phenotype and severity (8), features that provide support for the search for genetic loci that may be specific to pediatric disease. In addition, pediatric $\mathrm{CD}$ has a stronger familial component than the adult disease. Thus, targeting pediatric patients potentially provide additional power to identify molecular factors that contribute to pathogenesis of IBD, as illustrated by a transatlantic collaboration, which analyzed a cohort of 3426 childhood-onset IBD patients and identified five new loci associated with pediatric IBD (9).

The multi-drug resistance gene (MDR1) spans $209 \mathrm{~kb}$ and is located in the chromosome 7q21.1. It is composed of 28 exons and the cDNA is $4.5 \mathrm{~kb}$; thus, its coding region accounts for less than $5 \%$ of the total gene (10). The MDR1 gene codes for the P-glycoprotein 170 (P-gp170), a member of the adenosine triphosphate (ATP) binding cassette family. P-gp170 functions as a transmembrane efflux pump, thereby moving drugs from the intracellular to the extracellular domain (11). In the human gastrointestinal tract (GI), P-gp170 is found at high concentrations on apical surfaces of superficial columnar epithelial cells of the colon, distal small bowel, small biliary ductules, and small pancreatic ductules (12). The putative role of the P-gp170 in the GI tract is to decrease the absorption of endogenous and exogenous hydrophobic amphipathic toxins (13).

Evidences at functional and genetic levels point to MDR1 gene as an attractive candidate for the pathogenesis and therapeutic responses in patients with IBD (14). First, the MDR1 gene is present in a region of the human genome (7q21.1) that

\footnotetext{
'Department of Cellular and Molecular Biology, Cytokines and NO Synthases -Immunity and Pathogeny Team, Faculty of Biological Sciences, University of Sciences and Technology Houari Boumediene, Algiers, Algeria; ${ }^{2}$ Department of Pediatrics, Bologhine Hospital, Algiers, Algeria; ${ }^{3}$ Department of Pediatrics, University Hospital Center Mustapha Bacha, Algiers, Algeria; ${ }^{4}$ Department of Pediatrics, University Hospital Center Lamine Debaghine, Algiers, Algeria; ${ }^{5}$ Department of Pediatrics, University Hospital Center Issaad Hassani, Algiers, Algeria. Correspondence: Hamida Mesbah-Amroun (amrounhamida@yahoo.com)

Received 31 January 2016; accepted 21 June 2016; advance online publication 5 October 2016. doi:10.1038/pr.2016.163
} 


\section{Articles | Bouzidie tal.}

may harbor a disease gene involved in susceptibility to IBD (15). Second, MDR1a knockout mice develop a spontaneous colitis when maintained under specific pathogen-free conditions (16). The colitis is prevented and reversed by the administration of antibiotics, suggesting that the intestinal flora is necessary to initiate and perpetuate the inflammation. This model implies that loss of the xenobiotic efflux mechanism may promote the development of the colitis.

Three single-nucleotide polymorphisms (SNPs) in MDR1 have been most commonly studied: C3435T (rs1045642, synonymous), C1236T (rs1128503, synonymous), and G2677T/A (rs2032582, nonsynonymous).

Despite in vivo and in vitro studies, epidemiological evidence on MDR1 gene-associations with CD is largely inconsistent. Some studies have found associations between these variants and CD $(17,18)$; however, others have not $(19,20)$. Furthermore, two meta-analyses evaluating the potential association of C3435T and G2677A/T polymorphisms with IBD failed to show an association between these variants and $\mathrm{CD}$ $(21,22)$.

Pediatric $\mathrm{CD}$ has a distinct phenotype and the difference from adult $C D$ makes it a unique subgroup (8). It can thus be expected that MDR1-related genetic associations among pediatric $\mathrm{CD}$ patients may also differ from those observed among adults. Only two studies had investigated MDR1-gene associations with CD exclusively among pediatric $C D$ patients $(23,24)$. However, there is no report of the frequency distribution of the major allelic variants or haplotypes of MDR1 gene in the Algerian pediatric patients with CD. Therefore, we aimed to investigate the frequencies of MDR1 gene polymorphisms and haplotypes in an Algerian cohort of pediatric CD patients, and evaluate the association between those polymorphisms and the susceptibility to $\mathrm{CD}$ in the Algerian population. A genotype-phenotype analysis was performed by comparing the genotype distribution among specific CD phenotype vs. that among controls.

\section{RESULTS}

\section{Clinical Findings}

A total of 47 pediatric $C D$ cases and 100 controls were studied. The main clinical characteristics of patients included in the study are summarized in Table 1 . The mean age at diagnosis of the cases was $10.78 \pm 3.79 \mathrm{y}$. Most of the cases $(70.21 \%)$ belongs to A1b age category. $14.89 \%$ of the patients have a positive family history of CD, $27.65 \%$ had resective surgery, and $23.40 \%$ presents extra-intestinal manifestations (in particular articular). Analysis of the disease location revealed the following distribution: L3 \pm L4 (ileo-colonic with or without upper tract): $53.19 \%$, $\mathrm{L} 1 \pm \mathrm{L} 4$ (ileal with or without upper tract): $34.04 \%$ and L2 \pm L4 (colonic with or without upper tract): $12.76 \%$. No patient had disease affecting only the upper tract. Disease behavior was: B1 $\pm \mathrm{p}=61.70 \%, \mathrm{~B} 2 \pm \mathrm{p}=8.51 \%, \mathrm{~B} 3 \pm \mathrm{p}=6.38 \%, \mathrm{~B} 2 \mathrm{~B} 3 \pm \mathrm{p}=23.4 \%$.

\section{Genotype Analysis}

Distributions of MDR1 gene polymorphisms in CD patients and controls are shown in Table 2. Genotype frequencies for each SNP were in Hardy-Weinberg equilibrium. We detected no significant association with $\mathrm{CD}$ in our population for the MDR1 C3435T SNP in comparison to the control group, since the frequency of wild-type, heterozygous, and mutated homozygous genotypes did not differ among the two study groups.

In contrast, the risk allele $1236 \mathrm{~T}$ of the MDR1 gene was significantly increased in patients with CD (52.1 vs. $34.5 \%$ in controls, $P=0.005$; $\mathrm{OR}=2.067$; $\left.(\mathrm{CI})_{95 \%}=1.255-3.405\right)$. Homozygous and heterozygous carriers of the risk allele 1235T are significantly more frequent $(P=0.016)$ in the cases group (25.53 and 53.19\%, respectively) compared to the controls (14 and $41 \%$, respectively).

In CD patients, the frequency of G2677A/T mutation was significantly higher than in controls (allele T: 42.5 vs. $25 \%$; allele A: 2.12 vs. $0.5 \% ; P=0.0012$; $\mathrm{OR}=2.360 ;(\mathrm{CI})_{95 \%}=1.408$ 3.955). Homozygous and heterozygous carriers of the mutated allele $2677 \mathrm{~T}$ are significantly more frequent $(P=0.0022)$ in the cases group (17.02 and $51.06 \%$ respectively) compared to the controls ( 3 and $43 \%$ respectively).

We detected no homozygous carrier for the mutated allele $2677 \mathrm{~A}$ in the two studied groups.

\section{Haplotypes Calculation}

There are 12 possible haplotypes of the 3 MDR1 loci listed in Table 3 with their estimated frequencies. We did not observe a statistical significant difference when comparing the overall haplotypes distribution between $\mathrm{CD}$ patients and controls $(P=0.47)$. However, there was a weak evidence $(P=0.03)$ that the haplotype composed of only wild-type alleles at position $1236,2677,3435$ (CGC) is more frequent in the control group. In the $\mathrm{CD}$ patients group, this haplotype is in second position $(P=0.14)$ and the most frequent haplotype bears a mutated allele at position 1236 and wild type at position 2677 and 3435 (TGC).

Frequencies of haplotypes for MDR1 SNPs pairs are listed in Table 4. When comparing haplotype pairs between patients and controls, frequency of $3435 \mathrm{C}-2677 \mathrm{G}$ and $2677 \mathrm{G}-1236 \mathrm{C}$ pairs were significantly higher in the control group compared to patients ( $P=0.04$ and $P=0.01$ respectively). In the patients group, the frequency of 2677T-1236T haplotype was significantly higher compared to controls $(P=0.02)$.

\section{Phenotype/Genotype Analysis}

When the contribution of each SNP of the MDRlgene was investigated (Table 1), the strongest correlation could be ascribed to the C1236T SNP. In CD patients, the occurrence of one risk allele of C1236T SNP was significantly associated with resective surgery $(P=0.028, r=0.321)$. No correlation was found for age at diagnosis, family history, disease behavior, or extra-intestinal manifestations.

\section{DISCUSSION}

We carried out a case-control study to examine associations between the MDR1 gene and CD among Algerian pediatric patients. Furthermore, we compared the genotyping results with phenotypic characteristics of CD patients to identify a possible genotype-phenotype correlation. Of the three SNPs 


\section{MDR1 in pediatric Crohn's disease $\mid$ Articles}

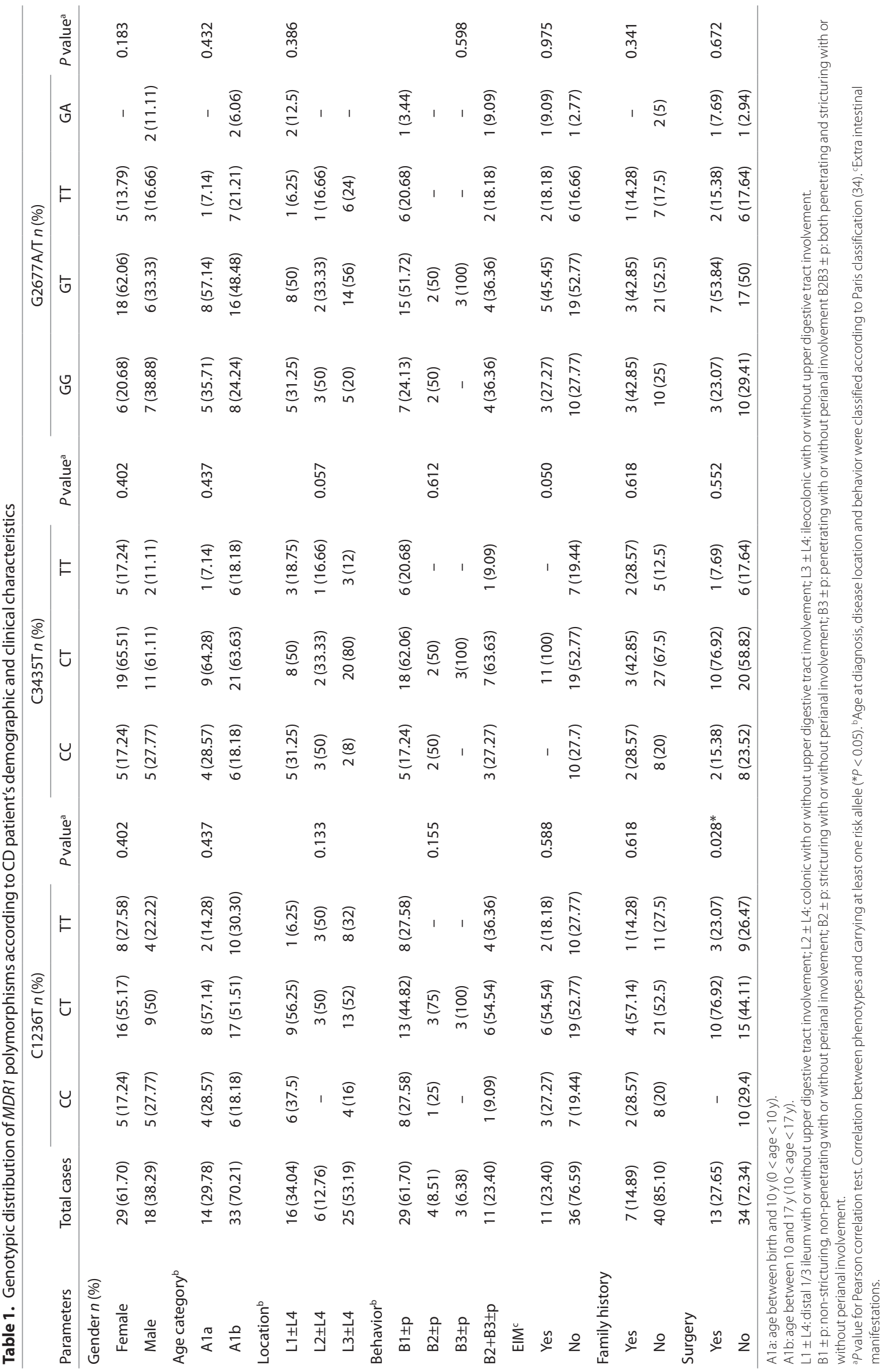




\section{Articles | Bouzidiet al.}

Table 2. MDR1 SNPs allele and genotype frequencies of early-onset CD patient's vs. control group

\begin{tabular}{|c|c|c|c|c|c|c|c|c|c|c|c|c|c|}
\hline SNP & $\begin{array}{l}\text { Amino } \\
\text { acid } \\
\text { change }\end{array}$ & Allele & $\begin{array}{c}\text { Controls } \\
n=100(\%)\end{array}$ & $\begin{array}{c}C D \\
n=47(\%)\end{array}$ & $P$ value $^{\mathrm{a}}$ & $\mathrm{Cl}_{95 \%}$ & OR & Genotype & Controls & $\begin{array}{c}\text { Pfor } \\
\text { HWE }^{\mathrm{b}}\end{array}$ & $C D$ & $\begin{array}{c}\text { Pfor } \\
\text { HWE }^{b}\end{array}$ & $P$ value $^{\mathrm{b}}$ \\
\hline rs1128503 & & C & $131(65.5)$ & $45(47.87)$ & 0.005 & $(1.255-3.405)$ & 2.067 & CC & $45(45)$ & 0.3533 & $10(21.27)$ & 0.6521 & 0.016 \\
\hline & & & & & & & & TT & $14(14)$ & & $12(25.53)$ & & \\
\hline rs2032582 & & G & $149(74.5)$ & $52(55.31)$ & & & & GG & $53(53)$ & & $13(27.65)$ & & \\
\hline & & A & $1(0.5)$ & $2(2.12)$ & $0.0012 * *$ & & 2.360 & TT & $3(3)$ & 0.1278 & $8(17.02)$ & 0.59 & $0.0022 * *$ \\
\hline & & & & & & & & AT & $1(1)$ & & - & & \\
\hline & & & & & & & & GA & - & & $2(4.25)$ & & \\
\hline & & & & & & & & $\mathrm{AA}$ & - & & - & & \\
\hline
\end{tabular}

$\mathrm{Cl}$, confidence interval; HWE: Hardy-Weinberg equilibrium; OR, odd ratio.

a $P$ values for Fisher's exact tests with a confidence interval of $95 \%\left({ }^{*} P<0.05,{ }^{* *} P<0.01\right)$. ${ }^{b} P$ values for Chi square test with a confidence interval of $95 \%\left({ }^{*} P<0.05,{ }^{* *} P<0.01\right)$.

Table 3. Comparison of haplotype frequencies for MDR1 $(1236 / 2677 / 3435)$ between CD patients and controls under the null hypothesis $(\mathrm{HO})$ assuming independence among all the loci

\begin{tabular}{lccc}
\hline & \multicolumn{2}{c}{ Haplotype frequencies } \\
\cline { 2 - 3 } Haplotype & $\begin{array}{c}\text { Controls (\%) } \\
\text { under H0 }\end{array}$ & CD (\%) under HO & P value \\
\hline CGC & 30.7424 & 14.0865 & $0.0311^{*}$ \\
CGT & 18.0551 & 12.3961 & 0.3873 \\
CAC & 0.2063 & 0.5418 & 0.779 \\
CAT & 0.1212 & 0.4768 & 0.6789 \\
CTC & 10.3163 & 10,8357 & 0.9239 \\
CTT & 6.0588 & 9.5355 & 0.4470 \\
TGC & 16,1926 & 15.3386 & 0.8946 \\
TGT & 9.5099 & 13.4980 & 0.4684 \\
TAC & 0.1087 & 0.5899 & 0.5958 \\
TAT & 0.0638 & 0.5192 & 0.5735 \\
TTC & 5.4338 & 11.7989 & 0.1724 \\
TTT & 3.1913 & 10.3831 & 0.0887 \\
Overall & & & 0.4700 \\
\hline
\end{tabular}

a $P$ values for Chi square test with a confidence interval of $95 \%(* P<0.05)$.

studied, associations with C1236T and G2677A/T SNPs were evident, whereas associations with C3435T were not significant. Moreover, our data suggest that the C1236T SNP correlates with the increased risk for surgery.

There is no published data on the epidemiology of CD in the Algerian pediatric population. However, our study provides first insights on the prevalence of the disease in Algiers as we enrolled 47 pediatric CD patients from 4 different pediatric departments during $2 \mathrm{y}$. Pediatric UC patients were also enrolled but the very small number of six patients did not allow us to conduct a case-control study.
Previous studies investigating MDR1 gene polymorphisms and its predisposition to $\mathrm{CD}$, have shown conflicting results. In a multicenter North American cohort with 444 IBD trios, Brant et al. investigated the G2677T/A and the C3435T SNPs (17). They reported a significant association of the G2677 variant with IBD confirmed by PDT (pedigree disequilibrium test) analysis, whereas no association was observed for the C3435T SNP. In contrast, Potocnik et al. reported no significant associations between both, C3435T and G2677T/A SNPs in a Slovenian cohort (25). However, they have documented positive associations of two other intronic SNPs (rs2235035 and rs1922242) with refractory CD. Furthermore, Urcelay et al. reported a significant association of the C3435Twild-type genotype in Spanish CD patients, but data were not in Hardy-Weinberg equilibrium (18).

In contrast, other studies failed to demonstrate an association between MDR1 variants and CD. In a German case-control study and in a large Scottish cohort, the authors reported a significant increase of the 3435 TT genotype and T-allelic frequencies in patients with UC but no association was seen with $\mathrm{CD}(26,27)$.

Reports in pediatric CD are limited. Only two studies had investigated associations of the MDR1 gene with CD exclusively among the pediatric population $(23,24)$. Cucchiara et al. have not found an association between the C3435T SNP and either predisposition to early onset $\mathrm{CD}$, clinical features, or response to medical therapy (23).The study by Krupoves et al. revealed that the SNP rs17327442 was significantly associated with an overall susceptibility to $\mathrm{CD}$. Analysis of the genotype-phenotype correlation indicated that two SNPs (rs10248420 and rs2032583) were significantly associated with colonic disease and that five SNPs (rs1128503, rs1202184, rs1202186, rs2091766, and rs2235046) were associated with structuring and/or penetrating disease (24). The reason for such variability of results in the literature is not clear. However, population ethnic heterogeneity and/or variability of exposure to environmental factors between populations might be plausible explanations. 
Table 4. Comparison of haplotype frequencies for pairs of MDR1 SNPs between CD patients and controls

\begin{tabular}{|c|c|c|c|c|c|c|c|c|c|c|c|}
\hline \multirow[b]{2}{*}{ Haplotype } & \multicolumn{2}{|c|}{ C3435T-G2677A/T } & \multirow[b]{2}{*}{$P$ value $^{\mathrm{a}}$} & & \multicolumn{2}{|c|}{ G2677A/T-C1236T } & \multirow[b]{2}{*}{$P$ value ${ }^{a}$} & & \multicolumn{2}{|c|}{ C3435T-C1236T } & \multirow[b]{2}{*}{$P$ value } \\
\hline & Controls & Patients & & & Controls & Patients & & & Controls & Patients & \\
\hline C-G & 46.93 & 29.42 & $0.0451^{*}$ & $\mathrm{G}-\mathrm{C}$ & 48.79 & 26.48 & $0.0108^{*}$ & $\mathrm{C}-\mathrm{C}$ & 41.26 & 25.46 & 0.0664 \\
\hline $\mathrm{C}-\mathrm{T}$ & 15.75 & 22.63 & 0.3124 & $\mathrm{~T}-\mathrm{C}$ & 16.37 & 20.37 & 0.5543 & $\mathrm{~T}-\mathrm{C}$ & 24.23 & 22.4 & 0.8082 \\
\hline T-T & 9.25 & 19.91 & 0.0708 & $\mathrm{~T}-\mathrm{T}$ & 8.62 & 22.18 & $0.0229^{*}$ & $\mathrm{~T}-\mathrm{T}$ & 12.76 & 24.4 & 0.0771 \\
\hline
\end{tabular}

a $P$ values for Chi square test with a confidence interval of $95 \%\left({ }^{*} P<0.05\right)$.

In our study, we described for the first time the distribution of MDR1 gene polymorphisms in a cohort composed of pediatric $\mathrm{CD}$ patients and healthy controls from Algeria.

Similar to the study by Cucchiara et al., our results indicated that the $\mathrm{C} 3435 \mathrm{~T}$ polymorphism is not a risk factor for $\mathrm{CD}$ in our cohort.

To the best of our knowledge, this is the first study reporting a significant association of the G2677A/T $(P=0.0012)$ SNPs with $\mathrm{CD}$ in the early onset population. Previous studies investigating the influence of the coding polymorphism G2677A/T on both mRNA and P-gp170 expression have reached conflicting results. Kim et al. reported an association of $2677 \mathrm{~T}$ allele with higher levels of P-gp170 expression (28). However, Kimchi-Sarfaty et al. documented no influence of this polymorphism on expression level and the intracellular localization of P-gp170 (29).

Moreover, our results indicated that the $\mathrm{C} 1236 \mathrm{~T}$ is a risk factor for $\mathrm{CD}$ in our cohort of Algerian pediatric CD patients $(P=0.005)$. This result is consistent with a study carried out in a Caucasian population from Canterbury, New Zealand. Based on stratification of all IBD patients with respect to age, the authors noted that individuals under $17 \mathrm{y}$ of age carrying the variant $1236 \mathrm{~T}$ allele were at an increased risk for CD development (30). The influence of C1236TSNP on the expression and P-gp170 activity still remains under investigation. In a study by Hemauer et al., it was indicated that in human placenta the 1236CT and 1236TT genotypes were related to a decreased P-gp170 expression in comparison to the wild-type 1236CC genotype (31). However, Goto et al. did not observe any correlation between the change in the intestinal mRNA expression of MDR1 or P-gp170 protein and the C1236T SNP in recipients of liver transplantation (32). It is suggested that MDR1 SNPs might exert their effect not by influencing expression level of P-gp170 but by altering the timing of co-translational folding and insertion of P-gp170 into the membrane, thereby altering the structure of substrate and inhibitor interaction sites (29). Consequently, the SNP might affect the affinity of theP-gp170 to toxins, which might lead to increased absorption of toxins in the gut and inflammation. Interestingly, the frequency of haplotype pairs $2677 \mathrm{~T}-1236 \mathrm{~T}$ was significantly higher in the patients group, compared to controls $(P=0.02)$, confirming thus their status as risk allele in the $\mathrm{CD}$ patients.
When comparing the genotype results with clinical characteristics of CD patients, the occurrence of one risk allele of C1236T SNP was moderately correlated with resective surgery $(P=0.028, r=0.321)$ which suggest a more aggressive course of the disease in carriers of at least one risk allele. Krupoves et al. analyzed children with CD $(12.1 \pm 3.5 \mathrm{y})$ from a Canadian population and observed a relationship between the C1236 polymorphism and stricture formation as well as penetrating disease $(P=0.02)(24)$. As far as we know, our study is the first report correlating the C1236T SNP with an increased risk for surgery, which could suggest a more severe disease course. In Table 1, we observe in one hand, that 1236TT genotype frequency is higher in the group of patients that did not have surgery. Following the outcome of this group of patients to see if they will need surgery would be interesting to confirm our results. In the other hand, none of patients with surgery had a 1236CC genotype which comfort our suggestion that 1236T allele is a risk allele for surgery.

It is important to acknowledge the strengths and limitations of the study prior to interpretation. First, because of the difficulty to get enrolled healthy children in our study, cases and controls are not matched regarding their age. However, enrolling adult controls does not impact our result because the matching factor (age in this case) is associated with disease (CD) but not exposure (MDR1 SNPs). Second, findings of association between MDR1 and pediatric CD disease susceptibility and risk for surgery should be treated with caution, as they were based on a small sample of subjects. Further studies with larger sample sizes are needed to fully confirm these findings. However, our result should get MDR1 SNPs, mainly $\mathrm{C} 1236 \mathrm{~T}$ and G2677A/T more interest, as there are very few studies investigating MDR1 SNPs in the pediatric CD population. To date, no meta-analysis investigating the association of $M D R 1$ with pediatric $\mathrm{CD}$ was performed. As a perspective, a meta-analysis, including our results would be interesting in order to cumulate and summarize the contribution of MDR1 gene contribution in pediatric CD patients. Moreover, further functional studies are required to address whether this SNP has an impact on the susceptibility to pediatric CD.

\section{Conclusion}

In this study, we have, for the first time assessed the association between the MDR1 polymorphisms and susceptibility to $C D$ 
in an Algerian pediatric cohort. Our results indicate that the C1236T and G2677A/T SNPs might represent relevant genetic factors/modifiers that may predispose towards pediatric CD. In order to better understand the relevance of these MDR1 gene polymorphisms in $\mathrm{CD}$, further functional studies are required to evaluate the P-gp170 gene expression and activity in the gastrointestinal tract.

\section{METHODS}

\section{Subjects}

To study associations between the MDR1 gene and CD, we carried out a case-control study.

The study was performed in accordance with the Declaration of Helsinki of the World Medical Association and was reviewed and approved by the Ethic Committee of the Agence Thématique de la Recherche Scientifique en Santé (ATRSS, ex ANDRS). Forty-seven unrelated pediatric $C D$ patients with a mean age of $10.78 \pm 3.79 \mathrm{y}$ were enrolled prospectively from four independent pediatric departments in Algiers, from January 2012 to June 2014. This cohort was not analyzed before in any IBD genetic studies. The diagnosis of CD was based on standard clinical, endoscopic, radiologic, and histopathological criteria (33). The Paris classification was used for CD disease type (34). In all of the patients, the following clinical features were recorded: family history, age at diagnosis, localization of CD (ileum, ileocolonic, colonic, upper GI tract), disease behavior (penetrating, stricturing, inflammatory (nonpenetrating and nonstricturing) presence of perianal fistulas, extraintestinal manifestations, and previous abdominal surgery (bowel restriction). The control group consisted of 100 unrelated healthy blood donors ( 59 female, 41 male) with a mean age of $23.6 \pm 5.2$ y without history of immune-mediated diseases. Prior to study enrollment, a voluntary written informed consent was obtained from all participants and legal guardians of patients under $16 \mathrm{y}$ of age. All groups studied originated from different regions of North Algeria and confirmed the Algerian origin of their parents and grandparents.

\section{Molecular Methods}

DNA was isolated from peripheral blood samples by phenol/chloroform extraction method according to standard protocols (35).

Three polymorphisms of the MDR1 (MIM*171050) gene including C3435T (exon 26, rs1045642), C1236T (exon 12, rs1128503), and G2677T/A (exon 21 rs2032582) were analyzed by PCR-RFLP method.

The following primers were used for PCR amplification.

For C3435T, forward primer: 5'-GATGTCTTGTGGGAGA GGGA- ${ }^{\prime}$ and reverse primer: 5'-GCATGTATGTTGGCCTCCTT-3' (36).

For C1236T, forward primer: 5'-TATCCTGTGTCTGTGAATT GCC-3' and reverse primer: 5'-CCTGACTCACCACACCAATG-3' (37).

For G2677A/T, forward primer: 5'-TGACAAACGTTAGGCTT AAATTACA- $3^{\prime}$ and reverse primer: $5^{\prime}$-AAGATTGCTTTGAGG AATGGTT-3' (38).

The PCR reactions were carried out using a T100 thermal cycler (Bio-Rad, Richmond, CA). PCR assays were performed using $200 \mathrm{ng}$ of genomic DNA, $10 \times$ buffer, $1.5 \mathrm{mmol} / \mathrm{l} \mathrm{MgCl}, 200 \mu \mathrm{mol} / \mathrm{l} \mathrm{dNTP}, 10$ pmol each primer and $1 \mathrm{U}$ Taq DNA polymerase (Promega, Madison, WI) in a final volume of $50 \mu \mathrm{l}$.

PCR conditions were as follows: predenaturation for $2 \mathrm{~min}$ at 96 ${ }^{\circ} \mathrm{C}$, followed by 35 cycles of denaturation for $30 \mathrm{~s}$ at $95^{\circ} \mathrm{C}$, annealing for $30 \mathrm{~s}$ by primer-specific temperature, primer extension for $30 \mathrm{~s}$ at $72{ }^{\circ} \mathrm{C}$, the final extension at $72^{\circ} \mathrm{C}$ for $5 \mathrm{~min}$. Annealing temperature for the C3435T, C1236T, and G2677T/A primers were 56, 60, and 63 ${ }^{\circ} \mathrm{C}$, respectively.

The restriction enzyme reactions were conducted following the manufacturer protocol. Twenty microliters of the PCR product were digested at $37{ }^{\circ} \mathrm{C}$ by MboI (EC: 3.1.21.4), (New England Biolabs, Ipswich, MA) and HaeIII (EC: 3.1.21.4), (Promega) for the polymorphisms C3435T and C1236T respectively. For the tri-allelic polymorphism G2677T/A, a double digestion was performed using RsaI and BseYI (New England Biolabs).

\section{Data Analysis}

Allele and genotype frequencies for MDR1 SNPs were assessed for deviation from the Hardy-Weinberg equilibrium using a chi-square test. The allele with the lower frequency among the controls was denoted as the "risk-conferring allele", whereas the common allele was denoted as the "wild-type" allele. Genotype and allele frequencies were compared between cases and controls using chi-square tests and Fisher's exact tests where appropriate. Odds ratios (OR) and 95\% confidence intervals $\left(\mathrm{CI}_{950}\right)$ were estimated. All statistical tests and $P$ values were two-tailed. Haplotypes frequency was calculated using the Estimated Haplotype (EH) program which is based on maximum likelihood calculations (39). The overall haplotype frequencies distribution and each single haplotype were compared between cases and controls using chi squared test.

Genotype-phenotype associations were measured using the Pearson correlation test. Statistical analyses were performed applying GraphPad prism (version 6.01 for Windows, GraphPad Software, La Jolla, CA).

\section{STATEMENT OF FINANCIAL SUPPORT}

This study was supported by the Agence Thématique de la Recherche Scientifique en Santé, (ATRSS, ex ANDRS) (PNR N³7-ANDRS-2011).

Disclosure: M-A.H. has received research funding from the Agence Thématique de la Recherche Scientifique en Santé (ATRSS, ex ANDRS). There is no relevant financial or non-financial relationship linked to products in this study. No conflict of interest, financial or other, exists.

\section{REFERENCES}

1. Kaser A, Zeissig S, Blumberg RS. Inflammatory bowel disease. Annu Rev Immunol 2010;28:573-621.

2. Ruemmele FM. Pediatric inflammatory bowel diseases: coming of age. Curr Opin Gastroenterol 2010;26:332-6.

3. Hugot JP, Chamaillard M, Zouali H, et al. Association of NOD2 leucine-rich repeat variants with susceptibility to Crohn's disease. Nature 2001;411:599-603.

4. Ogura $\mathrm{Y}$, Bonen DK, Inohara N, et al. A frameshift mutation in NOD2 associated with susceptibility to Crohn's disease. Nature 2001;411:603-6.

5. Duerr RH, Taylor KD, Brant SR, et al. A genome-wide association study identifies IL23R as an inflammatory bowel disease gene. Science 2006;314:1461-3.

6. Hampe J, Franke A, Rosenstiel P, et al. A genome-wide association scan of nonsynonymous SNPs identifies a susceptibility variant for Crohn disease in ATG16L1. Nat Genet 2007;39:207-11.

7. Van Limbergen J, Wilson DC, Satsangi J. The genetics of Crohn's disease. Annu Rev Genomics Hum Genet 2009;10:89-116.

8. Van Limbergen J, Russell RK, Drummond HE, et al. Definition of phenotypic characteristics of childhood-onset inflammatory bowel disease. Gastroenterology 2008;135:1114-22.

9. Imielinski M, Baldassano RN, Griffiths A, et al.; Western Regional Alliance for Pediatric IBD; International IBD Genetics Consortium; NIDDK IBD Genetics Consortium; Belgian-French IBD Consortium; Wellcome Trust Case Control Consortium. Common variants at five new loci associated with early-onset inflammatory bowel disease. Nat Genet 2009;41:1335-40.

10. Callen DF, Baker E, Simmers RN, Seshadri R, Roninson IB. Localization of the human multiple drug resistance gene, MDR1, to 7q21.1. Hum Genet 1987;77:142-4.

11. Sharom FJ. The P-glycoprotein multidrug transporter. Essays Biochem 2011;50:161-78.

12. Thiebaut F, Tsuruo T, Hamada H, Gottesman MM, Pastan I, Willingham MC. Cellular localization of the multidrug-resistance gene product P-glycoprotein in normal human tissues. Proc Natl Acad Sci USA 1987;84:7735-8.

13. Fricker G, Drewe J, Huwyler J, Gutmann H, Beglinger C. Relevance of p-glycoprotein for the enteral absorption of cyclosporin A: in vitro-in vivo correlation. Br J Pharmacol 1996;118:1841-7. 
14. Ho GT, Moodie FM, Satsangi J. Multidrug resistance 1 gene (P-glycoprotein 170): an important determinant in gastrointestinal disease? Gut 2003;52:759-66.

15. Satsangi J, Parkes M, Louis E, et al. Two stage genome-wide search in inflammatory bowel disease provides evidence for susceptibility loci on chromosomes 3, 7 and 12. Nat Genet 1996;14:199-202.

16. Panwala CM, Jones JC, Viney JL. A novel model of inflammatory bowel disease: mice deficient for the multiple drug resistance gene, mdrla, spontaneously develop colitis. J Immunol 1998;161:5733-44.

17. Brant SR, Panhuysen CI, Nicolae D, et al. MDR1 Ala893 polymorphism is associated with inflammatory bowel disease. Am J Hum Genet 2003;73:1282-92.

18. Urcelay E, Mendoza JL, Martín MC, et al. MDR1 gene: susceptibility in Spanish Crohn's disease and ulcerative colitis patients. Inflamm Bowel Dis 2006;12:33-7.

19. Palmieri O, Latiano A, Valvano R, et al. Multidrug resistance 1 gene polymorphisms are not associated with inflammatory bowel disease and response to therapy in Italian patients. Aliment Pharmacol Ther 2005;22:1129-38

20. Oostenbrug LE, Dijkstra G, Nolte IM, et al. Absence of association between the multidrug resistance (MDR1) gene and inflammatory bowel disease. Scand J Gastroenterol 2006;41:1174-82.

21. Onnie CM, Fisher SA, Pattni R, et al. Associations of allelic variants of the multidrug resistance gene (ABCB1 or MDR1) and inflammatory bowel disease and their effects on disease behavior: a case-control and metaanalysis study. Inflamm Bowel Dis 2006;12:263-71.

22. Annese V, Valvano MR, Palmieri O, Latiano A, Bossa F, Andriulli A. Multidrug resistance 1 gene in inflammatory bowel disease: a meta-analysis. World J Gastroenterol 2006;12:3636-44.

23. Cucchiara S, Latiano A, Palmieri O, et al.; Italian Society of Pediatric Gastroenterology and Nutrition. Polymorphisms of tumor necrosis factor-alpha but not MDR1 influence response to medical therapy in pediatric-onset inflammatory bowel disease. J Pediatr Gastroenterol Nutr 2007;44:171-9.

24. Krupoves A, Seidman EG, Mack D, et al. Associations between ABCB1/ MDR1 gene polymorphisms and Crohn's disease: a gene-wide study in a pediatric population. Inflamm Bowel Dis 2009;15:900-8.

25. Potocnik U, Ferkolj I, Glavac D, Dean M. Polymorphisms in multidrug resistance 1 (MDR1) gene are associated with refractory Crohn disease and ulcerative colitis. Genes Immun 2004;5:530-9.
26. Schwab M, Schaeffeler E, Marx C, et al. Association between the C3435T MDR1 gene polymorphism and susceptibility for ulcerative colitis. Gastroenterology 2003;124:26-33.

27. Ho GT, Nimmo ER, Tenesa A, et al. Allelic variations of the multidrug resistance gene determine susceptibility and disease behavior in ulcerative colitis. Gastroenterology 2005;128:288-96.

28. Kim RB, Leake BF, Choo EF, et al. Identification of functionally variant MDR1 alleles among European Americans and African Americans. Clin Pharmacol Ther 2001;70:189-99.

29. Kimchi-Sarfaty C, Oh JM, Kim IW, et al. A "silent" polymorphism in the MDR1 gene changes substrate specificity. Science 2007;315:525-8.

30. Huebner C, Browning BL, Petermann I, et al. Genetic analysis of MDR1 and inflammatory bowel disease reveals protective effect of heterozygous variants for ulcerative colitis. Inflamm Bowel Dis 2009;15:1784-93.

31. Hemauer SJ, Nanovskaya TN, Abdel-Rahman SZ, Patrikeeva SL, Hankins GD, Ahmed MS. Modulation of human placental P-glycoprotein expression and activity by MDR1 gene polymorphisms. Biochem Pharmacol 2010;79:921-5.

32. Goto M, Masuda S, Saito H, et al. C3435T polymorphism in the MDR1 gene affects the enterocyte expression level of CYP3A4 rather than Pgp in recipients of living-donor liver transplantation. Pharmacogenetics 2002;12:451-7.

33. Sands BE. From symptom to diagnosis: clinical distinctions among various forms of intestinal inflammation. Gastroenterology 2004;126:1518-32.

34. Levine A, Griffiths A, Markowitz J, et al. Pediatric modification of the Montreal classification for inflammatory bowel disease: the Paris classification. Inflamm Bowel Dis 2011;17:1314-21.

35. Sambrook J, Fritsch EF and Maniatis T. Molecular Cloning. Vol. 2. New York: Cold spring harbor laboratory press, 1989.

36. Sipeky C, Csongei V, Jaromi L, et al. Genetic variability and haplotype profile of MDR1 (ABCB1) in Roma and Hungarian population samples with a review of the literature. Drug Metab Pharmacokinet 2011;26:206-15.

37. Choi HJ, Cho HY, Ro H, et al. Polymorphisms of the MDR1 and MIF genes in children with nephrotic syndrome. Pediatr Nephrol 2011;26:1981-8.

38. Fajac A, Gligorov J, Rezai K, et al. Effect of ABCB1 C3435T polymorphism on docetaxel pharmacokinetics according to menopausal status in breast cancer patients. Br J Cancer 2010;103:560-6.

39. Excoffier L, Slatkin M. Maximum-likelihood estimation of molecular haplotype frequencies in a diploid population. Mol Biol Evol 1995;12: 921-7. 